\title{
Generalization of Finsler metrics on the product of Finsler manifolds
}

\author{
Akbar Sadighi $^{1} \cdot$ R. Chavosh Khatamy ${ }^{2} \cdot$ Megerdich Toomanian $^{1}$
}

Received: 21 February 2018 / Accepted: 25 September 2018 / Published online: 13 October 2018

(c) The Author(s) 2018

\begin{abstract}
In Riemannian manifolds, there exists a canonical Riemannian metric on the product of them (Lee in Riemannian geometry an introduction to curvature, Springer, New York, 1997). But at the product of Finsler manifolds, the canonical Finsler metric has not been defined. In this paper, we are going to study the product of Finsler manifolds and give a canonical Finsler metric on it.
\end{abstract}

Keywords Product of Finsler manifolds · Finsler manifolds · Minkowski structure

Mathematics Subject Classification 58B20 53C60

\section{Historical remarks}

The section brings a survey of Finsler geometry and product manifolds; a Finsler metric on a manifold is a collection of Minkowski norms $F_{x}$ in tangent space at $x$ such that $F_{x}$ varies smoothly in $x$.

In 1854, Reimann introduced the geometry based on the element of arc length

$d s=F\left(x^{1}, x^{2}, \ldots, x^{n}, \mathrm{~d} x^{1}, \mathrm{~d} x^{2}, \ldots, \mathrm{d} x^{n}\right)$.

where $F$ is positively homogeneous of degree 1 in $d x^{i}$ [2, 3]. For more than half a century, there had been no progress until P.Finsler introduced the Riemannian-Finsler geometry (Finsler geometry for short) in his thesis in 1918. A Finsler metric $F=F(x, y)$ is defined on tanget bundle $T M$, such that gives a for $x \in M$ by Minkowski norm [4-7],

$\left.F\right|_{T_{x} M}=F_{x}: T_{x} M \mapsto[0, \infty)$.

R. Chavosh Khatamy

r_chavosh@iaut.ac.ir

Akbar Sadighi

a.sadighi@kiau.ac.ir

Megerdich Toomanian

Megerdich.toomanian@kiau.ac.ir

1 Department of Mathematics, Karaj Branch, Islamic Azad University, Karaj, Iran

2 Department of Mathematics, Tabriz Branch, Islamic Azad University, Tabriz, Iran
Chern and Shen are defined product metric on Finsler spaces [8] as:

(1) For Finsler manifolds $\left(M_{1}, F_{1}\right)$ and $\left(M_{2}, F_{2}\right)$, $x=\left(x_{1}, x_{2}\right) \in M=M_{1} \times M_{2}$ and $y=\left(y_{1}, y_{2}\right) \in T_{\left(x_{1}, x_{2}\right)}\left(M_{1} \times\right.$ $\left.M_{2}\right)$, let $F: M=M_{1} \times M_{2} \mapsto[0, \infty)$ are defined by

$F(x, y):=\left\{\begin{array}{lll}F_{1}\left(x_{1}, y_{1}\right) & \text { if } & y=y_{1} \oplus 0 \\ F_{2}\left(x_{2}, y_{2}\right) & \text { if } & y=0 \oplus y_{2}\end{array}\right.$

Then $F$ is Finsler metric on $M_{1} \times M_{2}$, where $T_{x} M=T_{x_{1}} M_{1} \oplus T_{x_{2}} M_{2}$.

(2) For Riemannian manifolds $\left(M_{1}, g_{1}\right),\left(M_{2}, g_{2}\right)$ let $f:[0, \infty) \times[0, \infty) \mapsto[0, \infty)$ be a $c^{\infty}$ function satisfying:

$f(\lambda s, \lambda t)=\lambda f(s, t), \forall \lambda>0$, and $f(s, t)>0, \forall(s, t) \neq(0,0)$.

Then for all $\left(x_{1}, x_{2}\right) \in M_{1} \times M_{2}$ and $\left(y_{1}, y_{2}\right) \in T M_{1} \times T M_{2}$, $F$ defined function by

$F(x, y):=\sqrt{f\left(\left[g_{1}\left(x_{1}, y_{1}\right)\right]^{2},\left[g_{2}\left(x_{2}, y_{2}\right)\right]^{2}\right)}$

is a Finsler metric $M=M_{1} \times M_{2}$.

(3) Kozma et al. have defined a Twisted Products Finsler Manifolds [9] as:

Let $\left(M_{1}, F_{1}\right),\left(M_{2}, F_{2}\right)$ be two Finsler manifolds and $M=M_{1} \times M_{2}$, then for all $\left(x_{1}, x_{2}\right) \in M_{1} \times M_{2}$ and $\left(y_{1}, y_{2}\right) \in T_{\left(x_{1}, x_{2}\right)}\left(M_{1} \times M_{2}\right)-\{(0,0)\} \equiv\left(T_{x_{1}} M_{1}-\{0\}\right) \times$ $\left(T_{x_{2}} M_{2}-\{0\}\right)$ and $c^{\infty}$ function $f: M_{1} \times M_{2} \mapsto R^{+}$, the Twisted Products metric is defined by

$F\left(y_{1}, y_{2}\right):=\sqrt{F_{1}^{2}\left(x_{1}, y_{1}\right)+f^{2}\left(x_{1}, x_{2}\right) F_{2}^{2}\left(x_{2}, y_{2}\right)}$. 
$\left(y_{1}, y_{2}\right) \in T M_{1}-\{0\}$

In this paper, we are going to generalize a new product Finsler metric $F$ on $M=M_{1} \times M_{2}$, then we call $(M, F)$ as canonical product Finsler manifolds.

\section{Introduction and preliminaries}

We recall some definitions and fundamental results in Finsler geometry.

Definition 2.1 Let $V$ be a n-dimensional real vector space. A Minkowski norm on $V$ is a functional $F$ on $V$, which is smooth on $V-\{0\}$ and satisfies the following conditions:

(1) $F(u) \geq 0, \forall u \in V$;

(2) $F(\lambda u)=\lambda F(u), \forall \lambda>0, \forall u \in V$;

(3) for any basis $e_{1}, \ldots, e_{n}$ of $V$, write $F(y)=F\left(y^{1}, \ldots, y^{n}\right)$, $y=y^{j} e_{j}$. Then the Hessian matrix

$\left(g_{i j}\right):=\left(\left[\frac{1}{2} F^{2}\right]_{y^{i} y^{j}}\right)$

is positive definite at any point of $V-\{0\}$.

The pair $(V, F)$ is called Minkowski space.

Definition 2.2 Let $M$ be a (connected) smooth manifold. A Finsler metric on $M$ is a function $F: T M \rightarrow[0,+\infty)$ such that

(1) F is $C^{\infty}$ on the slit tangent bundle $T M-\{0\}$;

(2) The restriction of $F$ to any $T_{p} M, p \in M$ is a Minkowski norm.

The pair $(M, F)$ is called Finsler manifold or Finsler space.

Let $(M, F)$ be a Finsler space and $\left(x^{1}, \ldots, x^{n}\right)$ be a local coordinate system on an open subset $U$ of $M$. Then $\left\{\frac{\partial}{\partial x^{1}}, \ldots, \frac{\partial}{\partial x^{n}}\right\}$ form a basis for the tangent space at any point in $U$.

Theorem 2.3 (Euler's) Suppose a real-valued $H$ on $R^{n}$ is differentiable away from the origin of $R^{n}$. Then the following two statements are equivalent [5]

(I) $H$ is positively homogeneous of degree $r$. That is:

$$
H(\lambda y)=\lambda^{r} H(y) ; \quad \forall \lambda, \quad \lambda>0
$$

(II) The radial directional derivative of $\mathrm{H}$ is $r$ times $H$. Namely,

$$
y^{i} H_{y^{i}}(y)=r H(y) .
$$

Corollary 2.4 Let $F$ be positively homogeneous of degree 1 on $R^{n}$. By using Euler's theorem, we can show that:

(a) $y^{i} F_{y^{i}}=F$.

(b) $y^{i} F_{y^{i} y^{j}}=0$.

(c) $y^{k} F_{y^{i} y^{j} y^{k}}=-F_{y^{i}, y^{j}}$.

(d) $y^{l} F_{y^{i} y^{j} y^{k} y^{l}}=-2 F_{y^{i} y^{j} y^{k}}$.

Corollary 2.5 Let $(M, F)$ be a Finsler manifold. Then $F^{2}$ defined by:

$F_{p}^{2}(y)=\left(F_{p}(y)\right)^{2} ;$ for all $p \in M$ and $y \in T_{p} M$; is a positively homogeneous function of degree 2 on $T_{p} M$.

Corollary 2.6 Let $(M, F)$ be a Finsler manifolds. Then

$y^{i} \frac{\partial F^{2}(y)}{\partial y^{i}}=2(F(y))^{2}$.

For simplicity, we put $F_{1}\left(y_{1}\right), F_{2}\left(y_{2}\right)$; as $F_{p_{1}}\left(y_{1}\right), F_{p_{2}}\left(y_{2}\right)$, respectively. (i.e : $\left.F_{1}=F_{p_{1}}, F_{2}=F_{p_{2}}\right)$.

Proposition 2.7 Let $(M, F)$ be a Finsler manifold of dimension $n$. Then

$y^{i} \frac{\partial^{2}(F(y))^{2}}{\partial y^{i} \partial y^{j}}=\frac{\partial(F(y))^{2}}{\partial y^{j}}$.

Proof By definition and Corollary 2.6, we have:

$$
\begin{aligned}
& y^{i} \frac{\partial^{2}(F(y))^{2}}{\partial y^{i} \partial y^{j}}=\sum_{i=1}^{n} y^{i} \frac{\partial^{2}(F(y))^{2}}{\partial y^{i} \partial y^{j}}=\sum_{i=1}^{n} y^{i} \frac{\partial}{\partial y^{j}}\left(\frac{\partial(F(y))^{2}}{\partial y^{i}}\right) \\
& =\sum_{i=1_{i \neq j}}^{n} y^{i} \frac{\partial}{\partial y^{j}}\left(\frac{\partial(F(y))^{2}}{\partial y^{i}}\right)+y^{j} \frac{\partial}{\partial y^{j}}\left(\frac{\partial(F(y))^{2}}{\partial y^{j}}\right) \\
& =\sum_{i=1_{i \neq j}}^{n} \frac{\partial}{\partial y^{j}}\left(y^{i} \frac{\partial(F(y))^{2}}{\partial y^{i}}\right)+y^{j} \frac{\partial}{\partial y^{j}}\left(\frac{\partial(F(y))^{2}}{\partial y^{j}}\right) \\
& =\frac{\partial}{\partial y^{j}}\left(\sum_{i=1_{i \neq j}}^{n} y^{i} \frac{\partial(F(y))^{2}}{\partial y^{i}}\right)+y^{j} \frac{\partial}{\partial y^{j}}\left(\frac{\partial(F(y))^{2}}{\partial y^{j}}\right) \\
& =\frac{\partial}{\partial y^{j}}\left(\sum_{i=1}^{n} y^{i} \frac{\partial(F(y))^{2}}{\partial y^{i}}-y^{j} \frac{\partial(F(y))^{2}}{\partial y^{j}}\right)+y^{j} \frac{\partial}{\partial y^{j}}\left(\frac{\partial(F(y))^{2}}{\partial y^{j}}\right) \\
& =\frac{\partial}{\partial y^{j}}\left(2(F(y))^{2}-y^{j} \frac{\partial(F(y))^{2}}{\partial y^{j}}\right)+y^{j} \frac{\partial}{\partial y^{j}}\left(\frac{\partial(F(y))^{2}}{\partial y^{j}}\right)=\frac{\partial(F(y))^{2}}{\partial y^{j}} .
\end{aligned}
$$




\section{Minkowski structure on product of Finsler manifolds}

Theorem 3.1 Let $\left(M_{1}, F_{1}\right),\left(M_{2}, F_{2}\right)$ be Finsler Manifolds of dimensions $n$ and $m$, respectively. Suppose that $\left(p_{1}, p_{2}\right) \in M_{1} \times M_{2}$ and $F_{\left(p_{1}, p_{2}\right)}: T_{\left(p_{1}, p_{2}\right)}\left(M_{1} \times M_{2}\right) \rightarrow[0,+\infty)$ beafunction defined by: $F_{\left(p_{1}, p_{2}\right)}\left(y_{1}, y_{2}\right):=\left(F_{1} \oplus F_{2}\right)_{\left(p_{1}, p_{2}\right)}\left(y_{1}, y_{2}\right):=F_{1}\left(y_{1}\right)+F_{2}\left(y_{2}\right)$ $\forall\left(y_{1}, y_{2}\right) \in T_{\left(p_{1}, p_{2}\right)}\left(M_{1} \times M_{2}\right)$. Then,

(I) $\quad F_{\left(p_{1}, p_{2}\right)}\left(y_{1}, y_{2}\right) \geq 0, \quad \forall\left(y_{1}, y_{2}\right) \in T_{\left(p_{1}, p_{2}\right)}\left(M_{1}, M_{2}\right)$.

(II) $F_{\left(p_{1}, p_{2}\right)}\left(\lambda y_{1}, \lambda y_{2}\right)=\lambda F_{\left(p_{1}, p_{2}\right)}\left(y_{1}, y_{2}\right) ; \forall \lambda>0, \forall\left(y_{1}\right.$, $\left.y_{2}\right) \in T_{\left(p_{1}, p_{2}\right)}\left(M_{1}, M_{2}\right)$.

\section{Proof}

(I) Since for all $y_{1} \in T_{p_{1}} M_{1} ; F_{1}\left(y_{1}\right) \geq 0$ and for all $y_{2} \in T_{p_{2}} M_{2} ; F_{2}\left(y_{2}\right) \geq 0$, it follows that $F_{\left(p_{1}, p_{2}\right)}\left(y_{1}, y_{2}\right) \geq 0$.

(II) By the definition of Finsler metric, we have $\forall \lambda>0$, $F_{1}\left(\lambda y_{1}\right)=\lambda F_{1}\left(y_{1}\right), F_{2}\left(\lambda y_{2}\right)=\lambda F_{2}\left(y_{2}\right)$. It follows that $F_{\left(p_{1}, p_{2}\right)}\left(\lambda y_{1}, \lambda y_{2}\right)=\lambda F_{\left(p_{1}, p_{2}\right)}\left(y_{1}, y_{2}\right) ; \forall \lambda>0$.

Let us denote by

$y^{i}:=\left\{\begin{array}{rrrl}y_{1}^{i} & & 1 & \leq i \leq n \\ y_{2}^{i-n} & n+1 & \leq i \leq n+m\end{array}\right.$

and

$\left\{\begin{array}{cc}y_{1}^{i}=0 & n+1 \leq i \leq n+m \\ y_{2}^{i}=0 & 1 \leq i \leq n\end{array}\right.$.

Theorem 3.2 Let $\left(M_{1}, F_{1}\right),\left(M_{2}, F_{2}\right)$ be Finsler Manifolds of dimensions $n$ and $m$, respectively. Suppose that $\left(p_{1}, p_{2}\right) \in M_{1} \times M_{2}$ and $F_{\left(p_{1}, p_{2}\right)}: T_{\left(p_{1}, p_{2}\right)}\left(M_{1} \times M_{2}\right) \rightarrow[0,+\infty)$ be a function defined by:

$F_{\left(p_{1}, p_{2}\right)}\left(y_{1}, y_{2}\right):=\left(F_{1} \oplus F_{2}\right)_{\left(p_{1}, p_{2}\right)}\left(y_{1}, y_{2}\right):=F_{1}\left(y_{1}\right)+F_{2}\left(y_{2}\right)$, $\forall\left(y_{1}, y_{2}\right) \in T_{\left(p_{1}, p_{2}\right)}\left(M_{1}, M_{2}\right)$. Then the Hessian matrix $\left(g_{i j}\right):=\left(\left[\frac{1}{2} F^{2}\right]_{y^{i} y^{j}}\right)(y \neq 0)$ is positively definite.

Proof By the definition of positive definiteness in linear algebra, it is sufficient to show that:

$\forall y=\left(y_{1}, y_{2}\right) \in T_{\left(p_{1}, p_{2}\right)}\left(M_{1} \times M_{2}\right)-\{0\}$, we have

$[y]^{t}\left[g_{i j}\right][y]>0$. w h e r e $\quad y_{1}=\left(y_{1}^{1}, \ldots y_{1}^{n}\right) \in T_{p_{1}} M_{1}-\{0\} \quad$ a n d $y_{2}=\left(y_{2}^{1}, \ldots y_{2}^{m}\right) \in T_{p_{2}} M_{2}-\{0\} .[y]^{t}$ is transpose of matrix $[y]$. According to the definition of $F$, we have

$F^{2}(y)=\left[F_{1}\left(y_{1}\right)+F_{2}\left(y_{2}\right)\right]^{2}=F_{1}^{2}\left(y_{1}\right)+F_{2}^{2}\left(y_{2}\right)+2 F_{1}\left(y_{1}\right) F_{2}\left(y_{2}\right)$.

We now compute the Hessian matrix of component at slit tangent space

$T_{\left(p_{1}, p_{2}\right)}\left(M_{1} \times M_{2}\right)-\{0\}$ that is:

$g_{i j}:=\frac{1}{2} \frac{\partial^{2} F^{2}(y)}{\partial y^{i} \partial y^{j}}=\frac{1}{2} \frac{\partial^{2}}{\partial y^{i} \partial y^{j}}\left[F_{1}^{2}\left(y_{1}\right)+F_{2}^{2}\left(y_{2}\right)+2 F_{1}\left(y_{1}\right) F_{2}\left(y_{2}\right)\right]$.

For simplicity, we write $\left(g_{i j}\right)$ as, $\left(\begin{array}{ll}A_{11} & A_{12} \\ A_{21} & A_{22}\end{array}\right)$ where $A_{11}, A_{12}, A_{21}$ and $A_{22}$ are matrices, where.

$$
\begin{array}{ll}
\left(A_{11}\right)_{i j}=\frac{1}{2} \frac{\partial^{2} F^{2}(y)}{\partial y_{1}^{i} \partial y_{1}^{j}} ; \quad 1 \leq i, j \leq n, \\
\left(A_{12}\right)_{i j}=\frac{1}{2} \frac{\partial^{2} F^{2}(y)}{\partial y_{1}^{i} \partial y_{2}^{j}} ; \quad 1 \leq i \leq n, 1 \leq j \leq m \\
\left(A_{21}\right)_{i j}=\frac{1}{2} \frac{\partial^{2} F^{2}(y)}{\partial y_{2}^{i} \partial y_{1}^{j}} ; \quad 1 \leq i \leq m, 1 \leq j \leq n, \\
\left(A_{22}\right)_{i j}=\frac{1}{2} \frac{\partial^{2} F^{2}(y)}{\partial y_{2}^{i} \partial y_{2}^{j}} ; \quad 1 \leq i, j \leq m .
\end{array}
$$

By definition of $F$ and its partial derivatives, we have:

$$
\begin{aligned}
& \left(A_{11}\right)_{i j}=\frac{1}{2}\left(\frac{\partial^{2} F_{1}^{2}\left(y_{1}\right)}{\partial y_{1}^{i} \partial y_{1}^{j}}+2 F_{2}\left(y_{2}\right) \frac{\partial^{2} F_{1}\left(y_{1}\right)}{\partial y_{1}^{i} \partial y_{1}^{j}}\right) ; \quad 1 \leq i, j \leq n, \\
& \left(A_{12}\right)_{i j}=\frac{1}{2}\left(2 \frac{\partial F_{1}\left(y_{1}\right)}{\partial y_{1}^{i}} \times \frac{\partial F_{2}\left(y_{2}\right)}{\partial y_{2}^{j}}\right) ; \quad 1 \leq i \leq n, 1 \leq j \leq m, \\
& \left(A_{21}\right)_{i j}=\frac{1}{2}\left(2 \frac{\partial F_{1}\left(y_{1}\right)}{\partial y_{1}^{j}} \times \frac{\partial F_{2}\left(y_{2}\right)}{\partial y_{2}^{i}}\right) ; \quad 1 \leq j \leq n, 1 \leq i \leq m, \\
& \left(A_{22}\right)_{i j}=\frac{1}{2}\left(\frac{\partial^{2} F_{2}^{2}\left(y_{2}\right)}{\partial y_{2}^{i} \partial y_{2}^{j}}+2 F_{1}\left(y_{1}\right) \frac{\partial^{2} F_{2}\left(y_{2}\right)}{\partial y_{2}^{i} \partial y_{2}^{j}}\right) ; \quad 1 \leq i, j \leq m .
\end{aligned}
$$

It can be seen that: 


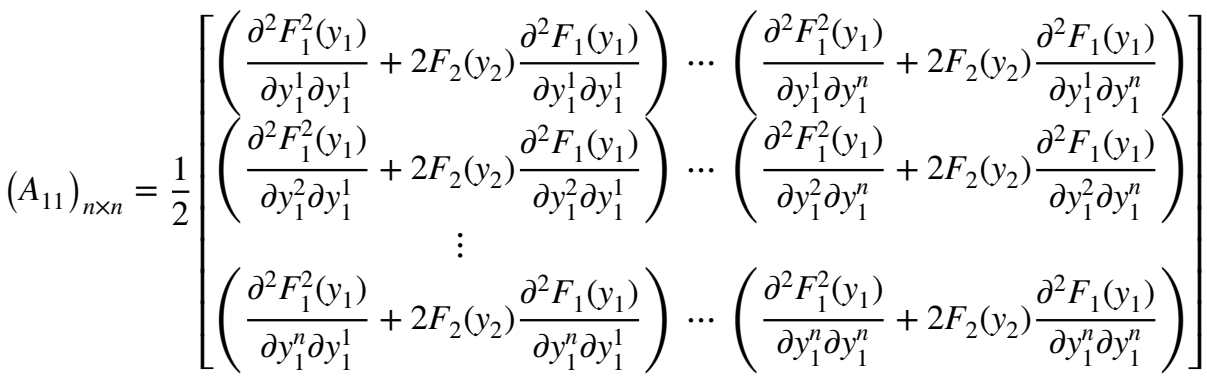

and

$$
\begin{array}{r}
\left(A_{12}\right)_{n \times m}=\frac{1}{2}\left[\begin{array}{c}
\left(2 \frac{\partial F_{1}\left(y_{1}\right)}{\partial y_{1}^{1}} \times \frac{\partial F_{2}\left(y_{2}\right)}{\partial y_{2}^{1}}\right) \cdots\left(2 \frac{\partial F_{1}\left(y_{1}\right)}{\partial y_{1}^{1}} \times \frac{\partial F_{2}\left(y_{2}\right)}{\partial y_{2}^{m}}\right) \\
\left(2 \frac{\partial F_{1}\left(y_{1}\right)}{\partial y_{1}^{2}} \times \frac{\partial F_{2}\left(y_{2}\right)}{\partial y_{2}^{1}}\right) \cdots\left(2 \frac{\partial F_{1}\left(y_{1}\right)}{\partial y_{1}^{2}} \times \frac{\partial F_{2}\left(y_{2}\right)}{\partial y_{2}^{m}}\right) \\
\left(2 \frac{\partial F_{1}\left(y_{1}\right)}{\partial y_{1}^{n}} \times \frac{\partial F_{2}\left(y_{2}\right)}{\partial y_{2}^{m}}\right) \cdots\left(2 \frac{\partial F_{1}\left(y_{1}\right)}{\partial y_{1}^{n}} \times \frac{\partial F_{2}\left(y_{n}\right)}{\partial y_{2}^{m}}\right)
\end{array}\right], \\
\left(A_{21}\right)_{m \times n}=\frac{1}{2}\left[\begin{array}{c}
\left(2 \frac{\partial F_{2}\left(y_{2}\right)}{\partial y_{2}^{1}} \times \frac{\partial F_{1}\left(y_{1}\right)}{\partial y_{1}^{1}}\right) \cdots\left(2 \frac{\partial F_{2}\left(y_{2}\right)}{\partial y_{2}^{1}} \times \frac{\partial F_{1}\left(y_{1}\right)}{\partial y_{1}^{n}}\right) \\
\left(2 \frac{\partial F_{2}\left(y_{2}\right)}{\partial y_{2}^{2}} \times \frac{\partial F_{1}\left(y_{1}\right)}{\partial y_{1}^{1}}\right) \cdots\left(2 \frac{\partial F_{2}\left(y_{2}\right)}{\partial y_{2}^{2}} \times \frac{\partial F_{1}\left(y_{1}\right)}{\partial y_{1}^{n}}\right) \\
\left(2 \frac{\partial F_{2}\left(y_{2}\right)}{\partial y_{2}^{m}} \times \frac{\partial F_{1}\left(y_{1}\right)}{\partial y_{1}^{1}}\right) \cdots\left(2 \frac{\partial F_{2}\left(y_{2}\right)}{\partial y_{2}^{m}} \times \frac{\partial F_{1}\left(y_{1}\right)}{\partial y_{1}^{n}}\right)
\end{array}\right]
\end{array}
$$

and

$\left(A_{22}\right)_{m \times}$

$$
\begin{aligned}
& {\left[\left(\frac{\partial^{2} F_{2}^{2}\left(y_{2}\right)}{\partial y_{2}^{1} \partial y_{2}^{1}}+2 F_{1}\left(y_{1}\right) \frac{\partial^{2} F_{2}\left(y_{2}\right)}{\partial y_{2}^{1} \partial y_{2}^{1}}\right) \cdots\left(\frac{\partial^{2} F_{2}^{2}\left(y_{2}\right)}{\partial y_{2}^{1} \partial y_{2}^{m}}+2 F_{1}\left(y_{1}\right) \frac{\partial^{2} F_{2}\left(y_{2}\right)}{\partial y_{2}^{1} \partial y_{2}^{m}}\right)\right]} \\
& =\frac{1}{2}\left[\begin{array}{ccc}
\left(\frac{\partial^{2} F_{2}^{2}\left(y_{2}\right)}{\partial y_{2}^{2} \partial y_{2}^{1}}+2 F_{1}\left(y_{1}\right) \frac{\partial^{2} F_{2}\left(y_{2}\right)}{\partial y_{2}^{2} \partial y_{2}^{1}}\right) \cdots\left(\frac{\partial^{2} F_{2}^{2}\left(y_{2}\right)}{\partial y_{2}^{2} \partial y_{2}^{m}}+2 F_{1}\left(y_{1}\right) \frac{\partial^{2} F_{2}\left(y_{2}\right)}{\partial y_{2}^{2} \partial y_{2}^{m}}\right) \\
\vdots \\
\left(\frac{\partial^{2} F_{2}^{2}\left(y_{2}\right)}{\partial y_{2}^{\prime \prime} \partial y_{2}^{1}}+2 F_{1}\left(y_{1}\right) \frac{\partial^{2} F_{2}\left(y_{2}\right)}{\partial y_{2}^{m} \partial y_{2}^{1}}\right) \cdots\left(\frac{\partial^{2} F_{2}^{2}\left(y_{2}\right)}{\partial y_{2}^{\prime m} \partial y_{2}^{m}}+2 F_{1}\left(y_{1}\right) \frac{\partial^{2} F_{2}\left(y_{2}\right)}{\partial y_{2}^{\prime \prime} \partial y_{2}^{m}}\right)
\end{array}\right]
\end{aligned}
$$

This give the Hessian matrix $\left(g_{i j}\right):=\left(\left[\frac{1}{2} F^{2}\right]_{y^{i} y^{j}}\right)=$ $\left(\begin{array}{ll}A_{11} & A_{12} \\ A_{12} & A_{22}\end{array}\right)$.

It is sufficient to show that: $\forall\left(y_{1}, y_{2}\right) \in T_{\left(p_{1}, p_{2}\right)}\left(M_{1} \times M_{2}\right)$ $-\{0\}$ we have:

$[y]^{t}\left[g_{i j}\right][y]>0 .\left[y_{1}^{1}, \ldots, y_{1}^{n}, y_{2}^{1}, \ldots, y_{2}^{m}\right]\left[g_{i j}\right]\left[\begin{array}{c}y_{1}^{1} \\ \vdots \\ y_{1}^{n} \\ y_{2}^{1} \\ \vdots \\ y_{2}^{m}\end{array}\right]=\left[y_{1}^{1}, \ldots, y_{1}^{n}, y_{2}^{1}, \ldots, y_{2}^{m}\right] \times \frac{1}{2}$

$$
\begin{aligned}
& {\left[\left(y_{1}^{i} \frac{\partial^{2}\left(F_{1}\left(y_{1}\right)\right)^{2}}{\partial y_{1}^{1} \partial y_{1}^{i}}\right)+2 F_{2}\left(y_{2}\right)\left(y_{1}^{i} \frac{\partial^{2} F_{1}\left(y_{1}\right)}{\partial y_{1}^{1} \partial y_{1}^{i}}\right)+2 \frac{\partial F_{1}\left(y_{1}\right)}{\partial y_{1}^{1}}\left(y_{2}^{j} \frac{\partial F_{2}\left(y_{2}\right)}{\partial y_{2}^{j}}\right)\right.} \\
& \left(y_{1}^{i} \frac{\partial^{2}\left(F_{1}\left(y_{1}\right)\right)^{2}}{\partial y_{1}^{2} \partial y_{1}^{i}}\right)+2 F_{2}\left(y_{2}\right)\left(y_{1}^{i} \frac{\partial^{2} F_{1}\left(y_{1}\right)}{\partial y_{1}^{2} \partial y_{1}^{i}}\right)+2 \frac{\partial F_{1}\left(y_{1}\right)}{\partial y_{1}^{2}}\left(y_{2}^{j} \frac{\partial F_{2}\left(y_{2}\right)}{\partial y_{2}^{j}}\right) \\
& \left(y_{1}^{i} \frac{\partial^{2}\left(F_{1}\left(y_{1}\right)\right)^{2}}{\partial y_{1}^{n} \partial y_{1}^{i}}\right)+2 F_{2}\left(y_{2}\right)\left(y_{1}^{i} \frac{\partial^{2} F_{1}\left(y_{1}\right)}{\partial y_{1}^{n} \partial y_{1}^{i}}\right)+2 \frac{\partial F_{1}\left(y_{1}\right)}{\partial y_{1}^{n}}\left(y_{2}^{j} \frac{\partial F_{2}\left(y_{2}\right)}{\partial y_{2}^{j}}\right) \\
& 2 \frac{\partial F_{2}\left(y_{2}\right)}{\partial y_{2}^{1}}\left(y_{1}^{i} \frac{\partial F_{1}\left(y_{1}\right)}{\partial y_{1}^{i}}\right)+\left(y_{2}^{j} \frac{\partial^{2}\left(F_{2}\left(y_{2}\right)\right)^{2}}{\partial y_{2}^{1} \partial y_{2}^{j}}\right)+2 F_{1}\left(y_{1}\right)\left(y_{2}^{j} \frac{\partial^{2} F_{2}\left(y_{2}\right)}{\partial y_{2}^{1} \partial y_{2}^{j}}\right) \\
& 2 \frac{\partial F_{2}\left(y_{2}\right)}{\partial y_{2}^{2}}\left(y_{1}^{i} \frac{\partial F_{1}\left(y_{1}\right)}{\partial y_{1}^{i}}\right)+\left(y_{2}^{j} \frac{\partial^{2}\left(F_{2}\left(y_{2}\right)\right)^{2}}{\partial y_{2}^{2} \partial y_{2}^{j}}\right)+2 F_{1}\left(y_{1}\right)\left(y_{2}^{j} \frac{\partial^{2} F_{2}\left(y_{2}\right)}{\partial y_{2}^{2} \partial y_{2}^{j}}\right) \\
& \left.2 \frac{\partial F_{2}\left(y_{2}\right)}{\partial y_{2}^{m}}\left(y_{1}^{i} \frac{\partial F_{1}\left(y_{1}\right)}{\partial y_{1}^{i}}\right)+\left(y_{2}^{j} \frac{\partial^{2}\left(F_{2}\left(y_{2}\right)\right)^{2}}{\partial y_{2}^{m} \partial y_{2}^{j}}\right)+2 F_{1}\left(y_{1}\right)\left(y_{2}^{j} \frac{\partial^{2} F_{2}\left(y_{2}\right)}{\partial y_{2}^{m} \partial y_{2}^{j}}\right)\right]
\end{aligned}
$$




$$
\begin{aligned}
& =\frac{1}{2} y_{1}^{1}\left[\left(y_{1}^{i} \frac{\partial^{2}\left(F_{1}\left(y_{1}\right)\right)^{2}}{\partial y_{1}^{1} \partial y_{1}^{i}}\right)+2 F_{2}\left(y_{2}\right)\left(y_{1}^{i} \frac{\partial^{2} F_{1}\left(y_{1}\right)}{\partial y_{1}^{1} \partial y_{1}^{i}}\right)+2 \frac{\partial F_{1}\left(y_{1}\right)}{\partial y_{1}^{1}}\left(y_{2}^{j} \frac{\partial F_{2}\left(y_{2}\right)}{\partial y_{2}^{j}}\right)\right] \\
& +\frac{1}{2} y_{1}^{2}\left[\left(y_{1}^{i} \frac{\partial^{2}\left(F_{1}\left(y_{1}\right)\right)^{2}}{\partial y_{1}^{2} \partial y_{1}^{i}}\right)+2 F_{2}\left(y_{2}\right)\left(y_{1}^{i} \frac{\partial^{2} F_{1}\left(y_{1}\right)}{\partial y_{1}^{2} \partial y_{1}^{i}}\right)+2 \frac{\partial F_{1}\left(y_{1}\right)}{\partial y_{1}^{2}}\left(y_{2}^{j} \frac{\partial F_{2}\left(y_{2}\right)}{\partial y_{2}^{j}}\right)\right] \\
& + \\
& \vdots \\
& +\frac{1}{2} y_{1}^{n}\left[\left(y_{1}^{i} \frac{\partial^{2}\left(F_{1}\left(y_{1}\right)\right)^{2}}{\partial y_{1}^{n} \partial y_{1}^{i}}\right)+2 F_{2}\left(y_{2}\right)\left(y_{1}^{i} \frac{\partial^{2} F_{1}\left(y_{1}\right)}{\partial y_{1}^{n} \partial y_{1}^{i}}\right)+2 \frac{\partial F_{1}\left(y_{1}\right)}{\partial y_{1}^{n}}\left(y_{2}^{j} \frac{\partial F_{2}\left(y_{2}\right)}{\partial y_{2}^{j}}\right)\right] \\
& +\frac{1}{2} y_{2}^{1}\left[2 \frac{\partial F_{2}\left(y_{2}\right)}{\partial y_{2}^{1}}\left(y_{1}^{i} \frac{\partial F_{1}\left(y_{1}\right)}{\partial y_{1}^{i}}\right)+\left(y_{2}^{j} \frac{\partial^{2}\left(F_{2}\left(y_{2}\right)\right)^{2}}{\partial y_{2}^{1} \partial y_{2}^{j}}\right)+2 F_{1}\left(y_{1}\right)\left(y_{2}^{j} \frac{\partial^{2} F_{2}\left(y_{2}\right)}{\partial y_{2}^{1} \partial y_{2}^{j}}\right)\right] \\
& +\frac{1}{2} y_{2}^{2}\left[2 \frac{\partial F_{2}\left(y_{2}\right)}{\partial y_{2}^{2}}\left(y_{1}^{i} \frac{\partial F_{1}\left(y_{1}\right)}{\partial y_{1}^{i}}\right)+\left(y_{2}^{j} \frac{\partial^{2}\left(F_{2}\left(y_{2}\right)\right)^{2}}{\partial y_{2}^{2} \partial y_{2}^{j}}\right)+2 F_{1}\left(y_{1}\right)\left(y_{2}^{j} \frac{\partial^{2} F_{2}\left(y_{2}\right)}{\partial y_{2}^{2} \partial y_{2}^{j}}\right)\right] \\
& + \\
& \vdots \\
& +\frac{1}{2} y_{2}^{m}\left[2 \frac{\partial F_{2}\left(y_{2}\right)}{\partial y_{2}^{m}}\left(y_{1}^{i} \frac{\partial F_{1}\left(y_{1}\right)}{\partial y_{1}^{i}}\right)+\left(y_{2}^{j} \frac{\partial^{2}\left(F_{2}\left(y_{2}\right)\right)^{2}}{\partial y_{2}^{m} \partial y_{2}^{j}}\right)+2 F_{1}\left(y_{1}\right)\left(y_{2}^{j} \frac{\partial^{2} F_{2}\left(y_{2}\right)}{\partial y_{2}^{m} \partial y_{2}^{j}}\right)\right] \\
& +\frac{1}{2} y_{1}^{k}\left[\left(y_{1}^{i} \frac{\partial^{2}\left(F_{1}\left(y_{1}\right)\right)^{2}}{\partial y_{1}^{k} \partial y_{1}^{i}}\right)+2 F_{2}\left(y_{2}\right)\left(y_{1}^{i} \frac{\partial^{2} F_{1}\left(y_{1}\right)}{\partial y_{1}^{k} \partial y_{1}^{i}}\right)+2 \frac{\partial F_{1}\left(y_{1}\right)}{\partial y_{1}^{k}}\left(y_{2}^{j} \frac{\partial F_{2}\left(y_{2}\right)}{\partial y_{2}^{j}}\right)\right] \\
& +\frac{1}{2} y_{2}^{h}\left[2 \frac{\partial F_{2}\left(y_{2}\right)}{\partial y_{2}^{h}}\left(y_{1}^{i} \frac{\partial F_{1}\left(y_{1}\right)}{\partial y_{1}^{i}}\right)+\left(y_{2}^{j} \frac{\partial^{2}\left(F_{2}\left(y_{2}\right)\right)^{2}}{\partial y_{2}^{h} \partial y_{2}^{j}}\right)+2 F_{1}\left(y_{1}\right)\left(y_{2}^{j} \frac{\partial^{2} F_{2}\left(y_{2}\right)}{\partial y_{2}^{h} \partial y_{2}^{j}}\right)\right]
\end{aligned}
$$

In the first expression by Corollaries 2.4 (b), and 2.5, 2.6 and Proposition 2.7, it follows that

$y_{1}^{i} \frac{\partial^{2} F_{1}\left(y_{1}\right)}{\partial y_{1}^{k} \partial y_{1}^{i}}=0, y_{2}^{j} \frac{\partial F_{2}\left(y_{2}\right)}{\partial y_{2}^{j}}=F_{2}\left(y_{2}\right), y_{1}^{i} \frac{\partial^{2}\left(F_{1}\left(y_{1}\right)\right)^{2}}{\partial y_{1}^{k} \partial y_{1}^{i}}=\frac{\partial\left(F_{1}\left(y_{1}\right)\right)^{2}}{\partial y_{1}^{k}}$.

In the second expression,

$y_{2}^{j} \frac{\partial^{2} F_{2}\left(y_{2}\right)}{\partial y_{2}^{h} \partial y_{2}^{j}}=0, \quad y_{1}^{i} \frac{\partial F_{1}\left(y_{1}\right)}{\partial y_{1}^{i}}=F_{1}\left(y_{1}\right)$

$y_{2}^{j} \frac{\partial^{2}\left(F_{2}\left(y_{2}\right)\right)^{2}}{\partial y_{2}^{h} \partial y_{2}^{j}}=\frac{\partial\left(F_{2}\left(y_{2}\right)\right)^{2}}{\partial y_{2}^{h}}$.

Then we will have

$$
\begin{aligned}
{[y]^{t}\left[g_{i j}\right][y]=} & \frac{1}{2} y_{1}^{k}\left[\frac{\partial\left(F_{1}\left(y_{1}\right)\right)^{2}}{\partial y_{1}^{k}}+2 \frac{\partial F_{1}\left(y_{1}\right)}{\partial y_{1}^{k}} F_{2}\left(y_{2}\right)\right] \\
& +\frac{1}{2} y_{2}^{h}\left[2 \frac{\partial F_{2}\left(y_{2}\right)}{\partial y_{2}^{h}} F_{1}\left(y_{1}\right)+\frac{\partial\left(F_{2}\left(y_{2}\right)\right)^{2}}{\partial y_{2}^{h}}\right] .
\end{aligned}
$$

Therefore $[y]^{t}\left[g_{i j}\right][y]=\left[F_{1}^{2}\left(y_{1}\right)+2 F_{1}\left(y_{1}\right) F_{2}\left(y_{2}\right)+F_{2}^{2}\left(y_{2}\right)\right]$ $=\left[F_{1}\left(y_{1}\right)+F_{2}\left(y_{2}\right)\right]^{2}$. It is clear that $\forall y=\left(y_{1}, y_{2}\right) \in T_{\left(p_{1}, p_{2}\right)}$ $\left(M_{1} \times M_{2}\right)-\{0\}$ we will have

$[y]^{t}\left[g_{i j}\right][y]>0$.

And since the Hessian matrix $\left(g_{i j}\right):=\left(\left[\frac{1}{2} F^{2}\right]_{y^{i} y^{j}}\right) ;(y \neq 0)$ is positive definite.

Corollary 3.3 Let $\left(M_{1}, F_{1}\right), \quad\left(M_{2}, F_{2}\right)$ be Finsler Manifolds of dimensions $n$ and $m$, respectively. Suppose that $\left(p_{1}, p_{2}\right) \in M_{1} \times M_{2}$ and $F_{\left(p_{1}, p_{2}\right)}: T_{\left(p_{1}, p_{2}\right)}\left(M_{1} \times M_{2}\right) \rightarrow[0,+\infty)$ be a function defined by:

$F_{\left(p_{1}, p_{2}\right)}\left(y_{1}, y_{2}\right)=\left(F_{1} \oplus F_{2}\right)_{\left(p_{1}, p_{2}\right)}\left(y_{1}, y_{2}\right)=F_{1}\left(y_{1}\right)+F_{2}\left(y_{2}\right)$,

$\forall\left(y_{1}, y_{2}\right) \in T_{\left(p_{1}, p_{2}\right)}\left(M_{1} \times M_{2}\right)$

Then $F_{\left(p_{1}, p_{2}\right)}: T_{\left(p_{1}, p_{2}\right)}\left(M_{1} \times M_{2}\right) \rightarrow[0,+\infty)$ is a Minkowski norm. 
Proof The proof is result of Theorems 3.1 and 3.2.

Theorem 3.4 Let $\left(M_{1}, F_{1}\right),\left(M_{2}, F_{2}\right)$ be Finsler manifolds of dimensions $n$ and $m$, respectively. Then the function $F: T\left(M_{1} \times M_{2}\right) \rightarrow[0,+\infty)$ defined by:

$F_{\left(p_{1}, p_{2}\right)}\left(y_{1}, y_{2}\right):=\left(F_{1} \oplus F_{2}\right)_{\left(p_{1}, p_{2}\right)}\left(y_{1}, y_{2}\right):=F_{1}\left(y_{1}\right)+F_{2}\left(y_{2}\right)$

$\forall\left(p_{1}, p_{2}\right) \in M_{1} \times M_{2}, \forall\left(y_{1}, y_{2}\right) \in T_{\left(p_{1}, p_{2}\right)}\left(M_{1} \times M_{2}\right)=T_{p_{1}} M_{1} \oplus T_{p_{2}} M_{2}$

is a $c^{\infty}$-function on the slit ' tangent bundle $T\left(M_{1} \times M_{2}-\{0\}\right.$.

Proof Since $F_{1}$ and $F_{2}$ are $c^{\infty}$-functions, it follows that vector function $\theta: T_{p_{1}} M_{1} \oplus T_{p_{2}} M_{2} \rightarrow[0,+\infty) \times[0,+\infty)$ defined by: $\theta\left(y_{1}, y_{2}\right)=\left(F_{1}\left(y_{1}\right), F_{2}\left(y_{2}\right)\right)$ is a $c^{\infty}$-function, and so $\lambda: R \times R \rightarrow R$ defined by $\lambda(s, t)=s+t$ is a $c^{\infty}$ -function. It follows that $F_{\left(p_{1}, p_{2}\right)}=\lambda o \theta$ is a $c^{\infty}$-function on $T\left(M_{1} \times M_{2}\right)-\{0\}$.

As the restriction of $F$ to any $T_{\left(p_{1}, p_{2}\right)}\left(M_{1} \times M_{2}\right)-\{0\}$ is $c^{\infty}$, we have $F$ is a $c^{\infty}$ function on the slit tangent bundle $T\left(M_{1} \times M_{2}\right)-\{0\}$.

\section{Finsler structure on product of Finsler manifolds}

Theorem 4.1 Let $\left(M_{1}, F_{1}\right),\left(M_{2}, F_{2}\right)$ be Finsler manifolds of dimensions $n$ and $m$, respectively. Then the function $F: T\left(M_{1} \times M_{2}\right) \rightarrow[0,+\infty)$ defined by:

$\forall\left(p_{1}, p_{2}\right) \in M_{1} \times M_{2}, \forall\left(y_{1}, y_{2}\right) \in T_{\left(p_{1}, p_{2}\right)}\left(M_{1} \times M_{2}\right)=T_{p_{1}} M_{1} \oplus T_{p_{2}} M_{2}$

$F_{\left(p_{1}, p_{2}\right)}\left(y_{1}, y_{2}\right):=\left(F_{1} \oplus F_{2}\right)_{\left(p_{1}, p_{2}\right)}\left(y_{1}, y_{2}\right):=F_{1}\left(y_{1}\right)+F_{2}\left(y_{2}\right)$

is a Finsler metric on $M_{1} \times M_{2}$.

Proof By the Corollary 3.3, the restriction of $F$ to $T_{\left(p_{1}, p_{2}\right)}\left(M_{1} \times M_{2}\right), \forall\left(p_{1}, p_{2}\right) \in M_{1} \times M_{2}$ is s Minkowski norm, and according to the Theorem $3.4 F$ is $c^{\infty}$ function on the slit tangent bundle $T\left(M_{1} \times M_{2}\right)-\{0\}$. Therefore $F$ is a Finsler metric on $M_{1} \times M_{2}$.
Definition 4.2 Let $\left(M_{1}, F_{1}\right),\left(M_{2}, F_{2}\right)$ be Finsler manifolds of dimensions $n$ and $m$, respectively. Then Finsler metric $F=F_{1}+F_{2}$ on $M_{1} \times M_{2}$ is called canonical product Finsler metric.

Open Access This article is distributed under the terms of the Creative Commons Attribution 4.0 International License (http://creativecommons.org/licenses/by/4.0/), which permits unrestricted use, distribution, and reproduction in any medium, provided you give appropriate credit to the original author(s) and the source, provide a link to the Creative Commons license, and indicate if changes were made.

\section{References}

1. Lee, J.M.: Riemannian Geometry an Introduction to Curvature. Springer, New York (1997)

2. Tu, L.W.: An Introduction to Manifolds. Springer, New York (2011)

3. Do Carmo, M.: Riemannian Geometry. Birkkauser, Boston (1997)

4. Bao, D., Chern, S.S., Shen, Z.: An Introduction to Riemann-Finsler Geometry. Springer, New York (2000)

5. Bao, D., Chern, S.S.: On a notable connection in Finsler geometry. Houst. J. Math. 19, 135-180 (1993)

6. Zixin, H., Deng, S.: On symmetric Finsler space. Isr. J. Math. 162, 197-219 (2007)

7. Toomanian, M., Khatamy, R.C.: Existence of homogeneous vector on the space of the tangent bundle. Acta. Math. Hung. 116(4), 285-294 (2007)

8. Shen, Z., Chern, S.S.: Riemann-Finsler Geometry. World Science, Singapore (2005)

9. Kozma, L., Peter, I.R., Shimada, H.: On the twisted product of Finsler manifolds. Elsevier Rep. Math. Phys. 57(3), 375-383 (2006)

Publisher's Note Springer Nature remains neutral with regard to jurisdictional claims in published maps and institutional affiliations. 